\section{Sensory Quality Attributes of Butterhead Lettuce Cultivars Grown in Arid Conditions}

\author{
Abdulla A. Alsadon \\ Department of Plant Production, College of Agriculture, King Saud University, \\ P.O. Box 2460, Riyadh 11451, Saudi Arabia
}

Additional index words. Lactuca sativa, defects, appearance, firmness

\begin{abstract}
Six butterhead lettuce (Lactuca sativa L.) cultivars-'Sorbon' (L2130), 'Donatan' (L2694), 'Jessy', 'Aramir', 'Florian', and 'Suzan' - w e r e grown in the central region of Saudi Arabia. Following harvest and yield assessment, individual heads from each cultivar were selected randomly for subjective sensory evaluation. Among the quality attributes evaluated were color, appearance, head firmness, texture, flavor, and freedom from defects. Attributes were highly correlated with overall quality for each cultivar. 'Florian' attributes were ranked high by members of an evaluation panel. In addition, 'Florian' plants were among the highest yielding cultivars. 'Florian' is recommended for its yield and quality features.
\end{abstract}

Sensory evaluation of horticultural crops depends on and is affected by diverse factors (Lipton, 1980; Watada, 1980). The present study evaluated the sensory attributes of six cultivars of lettuce grown in fields for two seasons (1989-90 and 1990-91) in central Saudi Arabia. The average temperature and relative humidity during winter growing seasons are shown in Table 1. The experiments were conducted at the Agricultural Research and Experiment Station at Dirab, Riyadh, Saudi Arabia. Cultural practices were uniform among cultivars and during both seasons. The cultivars were 'Sorbon' (L2130), 'Donatan' (L2694), 'Jessy', 'Aramir', 'Florian', and 'Suzan'. Seeds were sown in JV7 pellets (Jiffy Products, Norway) and kept in the greenhouse for 35 days before transplanting on $6 \mathrm{Dec}$. 1989 and 20 Dec. 1990 for the first and second seasons, respectively. Yields and stability parameters were reported previously by Alsadon et al. (1991).

At harvest, 28 heads from each cultivar were selected randomly, washed, air-dried, wrapped, and distributed to 28 untrained panel members for evaluation. Traits evaluated were color, appearance (shape and bolting), firmness, texture, flavor, defects (mechanical damage, decay, wilting, and tip bum), and overall quality. Evaluation was based on a scale of $1=$ very poor, $2=$ poor, $3=$ fair, $4=$ good, and $5=$ very good. The evaluation system was adapted from Kader et al. (1973).

Received for publication 13 Dec. 1991. Accepted for publication 23 July 1992. Support from the Agricultural Research and Experiment Station at Dirab, Riyadh, Saudi Arabia, is gratefully acknowledged. The cost of publishing this paper was defrayed in part by the payment of page charges. Under postal regulations, this paper therefore mus be hereby marked advertisement solely to indicate this fact.
Table 1. Temperature and relative humidity data during $1989-90$ and $1990-91$ growing seasons. $^{z}$

\begin{tabular}{|c|c|c|c|c|c|c|c|c|}
\hline \multirow[b]{3}{*}{ Period } & \multicolumn{4}{|c|}{ Temp ( $\left.{ }^{\circ} \mathrm{C}\right)$} & \multicolumn{4}{|c|}{ Relative humidity ( \%) } \\
\hline & \multicolumn{2}{|c|}{$\operatorname{Max}$} & \multicolumn{2}{|c|}{ Min } & \multicolumn{2}{|c|}{ Max } & \multicolumn{2}{|c|}{ Min } \\
\hline & Mean & Range & Mean & Range & Mean & Range & Mean & Range \\
\hline \multicolumn{9}{|c|}{ 1989-90 Season } \\
\hline Dec. 1989 & 22 & $16-31$ & 9 & $2-20$ & 62 & $28-73$ & 9 & $3-17$ \\
\hline Jan. 1990 & 19 & $12-29$ & 6 & $0-12$ & 80 & $6-98$ & 34 & $12-60$ \\
\hline Fcb. 1990 & 22 & $14-30$ & 8 & $0-15$ & 74 & $8-96$ & 33 & $19-74$ \\
\hline \multicolumn{9}{|c|}{ 1990-91 Season } \\
\hline Dec. 1990 & 27 & $14-30$ & 6 & $(-2)-10$ & 84 & $65-95$ & 26 & $14-45$ \\
\hline Jan. 1991 & 20 & $9-29$ & 7 & $(-3)-15$ & 87 & 59-95 & 38 & $18-70$ \\
\hline Feb. 1991 & 24 & $19-35$ & 9 & $1-17$ & 64 & $27-95$ & 34 & $17-58$ \\
\hline
\end{tabular}

${ }^{\mathrm{Z}}$ Data were collected by the weather station of the Agricultural Research and Experiment Station, College of Agriculture, at Dirab, Riyadh, Saudi Arabia. Data cover the period from transplanting in the open field until harvest (4 Feb. 1990 and 25 Feb. 1991, respectively).

Table 2. Correlation coefficients between the overall quality and other attributes of butterhead lettuce cultivars during 1989-90 and 1990-91 growing seasons.

\begin{tabular}{llccccc}
\hline & \multicolumn{5}{c}{ Attribute } \\
\cline { 2 - 7 } Cultivar & Color & Appearance & Firmness & Texture & Flavor & Defects \\
\hline Sorbon & $0.802^{* *}$ & $0.678^{* *}$ & $0.750^{* *}$ & $0.312^{\text {ss }}$ & $0.710^{* *}$ & $0.524^{* *}$ \\
Donatan & $0.754^{* *}$ & $0.864^{* *}$ & $0.727^{* *}$ & $0.630^{* *}$ & $0.747^{* *}$ & $0.729^{* *}$ \\
Jessy & $0.693^{* *}$ & $0.748^{* *}$ & $0.751^{* *}$ & $0.834^{* *}$ & $0.917^{* *}$ & $0.606^{* *}$ \\
Aramir & $0.707^{* *}$ & $0.648^{* *}$ & $0.682^{* *}$ & $0.619^{* *}$ & $0.743^{* *}$ & $0.623^{* *}$ \\
Florian & $0.747^{* *}$ & $0.818^{* *}$ & $0.728^{* *}$ & $0.731^{* *}$ & $\mathbf{0 . 7 1 3}^{* *}$ & $0.659^{* *}$ \\
Suzan & $0.611^{* *}$ & $0.618^{* *}$ & $0.604^{* *}$ & $0.693^{* *}$ & $0.415^{*}$ & $\mathbf{0 . 4 7 9 ^ { * * }}$ \\
\hline
\end{tabular}

Ns,",**N"Nonsignificant or significant at $P=0.05$ or 0.01 , respectively.

Table 3. Statistics of sensory ranking scores $(\bar{X}=$ ranking means from 28 panelists $)$.

\begin{tabular}{lccccccr}
\hline \hline & & \multicolumn{7}{c}{ Cultivar } \\
\cline { 3 - 8 } Attribute & Statistic & Sorbon & Donatan & Jessy & Aramir & Florian & Suzan \\
\hline Color & $\overline{\mathrm{X}}$ & 3.21 & 3.28 & 3.50 & 3.21 & 4.17 & 3.14 \\
& $\mathrm{SD}$ & 1.19 & 1.08 & 1.38 & 1.13 & 1.02 & 1.26 \\
Appearance & $\overline{\mathrm{X}}$ & 3.07 & 3.25 & 3.00 & 2.92 & 3.35 & 2.61 \\
& $\mathrm{SD}$ & 1.02 & 1.07 & 1.08 & 1.05 & 1.36 & 1.06 \\
Firmness & $\overline{\mathrm{X}}$ & 3.21 & 3.53 & 3.00 & 3.25 & 3.42 & 3.00 \\
& $\mathrm{SD}$ & 1.31 & 1.07 & 1.12 & 1.10 & 1.39 & 1.12 \\
Texture & $\overline{\mathrm{X}}$ & 3.25 & 3.75 & 3.46 & 3.39 & 3.64 & 3.39 \\
& $\mathrm{SD}$ & 1.04 & 0.79 & 1.17 & 1.06 & 1.06 & 1.16 \\
Flavor & $\overline{\mathrm{X}}$ & 3.35 & 2.93 & 3.39 & 3.39 & 3.96 & 3.46 \\
& $\mathrm{SD}$ & 1.02 & 1.18 & 1.16 & 1.10 & 0.96 & 1.17 \\
Freedom from & & & & & & & \\
defects & $\overline{\mathrm{X}}$ & 3.18 & 2.82 & 3.60 & 3.25 & 3.82 & 3.03 \\
& $\mathrm{SD}$ & 1.22 & $\mathbf{1 . 1 8}$ & 1.06 & 1.17 & 1.12 & 1.10 \\
Overall quality & $\mathrm{X}$ & 3.21 & 3.26 & 3.32 & 3.23 & 3.73 & 3.10 \\
& $\mathrm{SD}$ & 0.72 & 0.79 & 0.85 & 0.74 & 0.85 & 0.65 \\
\hline
\end{tabular}


Table 4. Frequency percentage of individual attribute rankings and overall quality of six butterhead lettuce cultivars.

\begin{tabular}{|c|c|c|c|c|c|c|c|}
\hline \multirow[b]{2}{*}{ Attribute } & \multirow[b]{2}{*}{ Ranking $^{z}$} & \multicolumn{6}{|c|}{ Cultivar } \\
\hline & & Sorbon & Donatan & Jessy & Aramir & Florian & Suzan \\
\hline \multicolumn{8}{|c|}{ Percent } \\
\hline \multirow[t]{5}{*}{ Color } & 1 & 4 & 4 & 4 & 7 & 0 & 11 \\
\hline & 2 & 32 & 25 & 18 & 21 & 11 & 25 \\
\hline & 3 & 21 & 21 & 25 & 25 & 11 & 18 \\
\hline & 4 & 25 & 39 & 32 & 36 & 29 & 32 \\
\hline & 5 & 18 & 11 & 21 & 11 & 50 & 14 \\
\hline \multirow[t]{5}{*}{ Visual quality } & 1 & 7 & 0 & 11 & 4 & 7 & 18 \\
\hline & 2 & 21 & 32 & 21 & 40 & 29 & 29 \\
\hline & 3 & 32 & 25 & 29 & 25 & 14 & 29 \\
\hline & 4 & 36 & 29 & 36 & 25 & 21 & 25 \\
\hline & 5 & 4 & 14 & 4 & 7 & 29 & 0 \\
\hline \multirow[t]{5}{*}{ Firmness } & 1 & 14 & 0 & 7 & 4 & 14 & 11 \\
\hline & 2 & 18 & 21 & 36 & 29 & 11 & 18 \\
\hline & 3 & 14 & 25 & 11 & 18 & 21 & 43 \\
\hline & 4 & 40 & 32 & 43 & 39 & 25 & 18 \\
\hline & 5 & 14 & 21 & 4 & 11 & 29 & 11 \\
\hline \multirow[t]{5}{*}{ Texture } & 1 & 4 & 0 & 4 & 4 & 0 & 4 \\
\hline & 2 & 21 & 11 & 25 & 18 & 21 & 25 \\
\hline & 3 & 32 & 14 & 11 & 29 & 14 & 18 \\
\hline & 4 & 32 & 64 & 43 & 36 & 43 & 36 \\
\hline & 5 & 11 & 11 & 18 & 14 & 21 & 18 \\
\hline \multirow[t]{5}{*}{ Flavor } & 1 & 4 & 11 & 4 & 7 & 0 & 11 \\
\hline & 2 & 18 & 32 & 25 & 14 & 11 & 7 \\
\hline & 3 & 29 & 18 & 18 & 21 & 14 & 21 \\
\hline & 4 & 39 & 32 & 36 & 46 & 43 & 46 \\
\hline & 5 & 11 & 7 & 18 & 11 & 32 & 14 \\
\hline \multirow[t]{5}{*}{ Freedom from def } & ects 1 & 7 & 11 & 4 & 4 & 4 & 7 \\
\hline & 2 & 32 & 39 & 14 & 29 & 11 & 32 \\
\hline & 3 & 7 & 14 & 18 & 25 & 18 & 14 \\
\hline & 4 & 43 & 29 & 46 & 25 & 36 & 43 \\
\hline & 5 & 11 & 7 & 18 & 18 & 32 & 4 \\
\hline \multirow[t]{5}{*}{ Overall quality } & 1 & 7 & 0 & 4 & 4 & 0 & 0 \\
\hline & 2 & 11 & 18 & 18 & 7 & 11 & 14 \\
\hline & 3 & 32 & 36 & 25 & 40 & 14 & 36 \\
\hline & 4 & 50 & 36 & 39 & 43 & 36 & 46 \\
\hline & 5 & 0 & 11 & 14 & 7 & 39 & 4 \\
\hline
\end{tabular}

${ }^{2}$ Ranking scale: 1 = very poor, $2=$ poor, $3=$ fair, $4=$ good, $5=$ very good. cultivars. The results were similar for texture, flavor, and overall quality. 'Florian' produces well-filled heads and tolerates unfavorable field conditions well (Alsadon et al., 1991).

\section{Literature Cited}

Alsadon, A.A., A.A. Gad, and S.O. Khalil. 1991. Evaluation of crisp head lettuce cultivars: 1 . Yield stability parameters. Alex. J. Agr. Res. 36:219-234. [Author note: Title should have referred to butterhead lettuce rather than crisphead lettuce.]

Kader, A.A., W.J. Lipton, and L.L. Morris. 1973. System for scoring quality of harvested lettuce. HortScience 5:408-409.

Lipton, W.J. 1980. Interpretation of quality evaluations of horticultural crops. HortScience 15:64 66

Ray, A.A. and J.P. Sall. 1982. SAS user's guide: Statistics. SAS Inst. Statistical Analysis System, Cary, N.C.

Ryder, E.J. 1979. Leafy salad vegetables. A VI, Westport, Conn.

Watada, A.E. 1980. Quality evaluation of horticultural crops: The problems. HortScience 15:47. 